\title{
Case 63: More Recurrent Seroma
}

\author{
E. Charles R. Lee and E. Antonio Mangubat
}

\subsection{Submitted by Charles Lee: April 10, 2008}

I had a similar problem (referring to Case 62 Serous Discharge, Chap. 147).

A 35-year-old with subglandular textured silicone implants that went well for 1 month, then she complained of swelling of the right breast and discharge from the wound. She has gone back to the UK to see her parents and I have discussed her with Tony (Erian) and we both agreed to sit on this, as there was no obvious infection.

However she had a cosmetic surgeon in the UK remove the right implant (after advice from me) and the same surgeon reinserted the same type of implant after 3 months and the swelling recurred in 2 weeks with discharge from the wound and the implant had to be removed again.

We (the UK surgeon and myself) are now both wondering what to do next. Please help. The left breast continues to behave well and the patient is extremely pleased with it.

I have attached a picture taken by the patient 1 month following the initial surgery (Fig. 148.1).

Please note; no cultures of the fluid were done while she was in the UK.

\section{Mangubat}

I would switch to smooth implants. I believe textured implants have been reported to cause seromas more often than smooth.

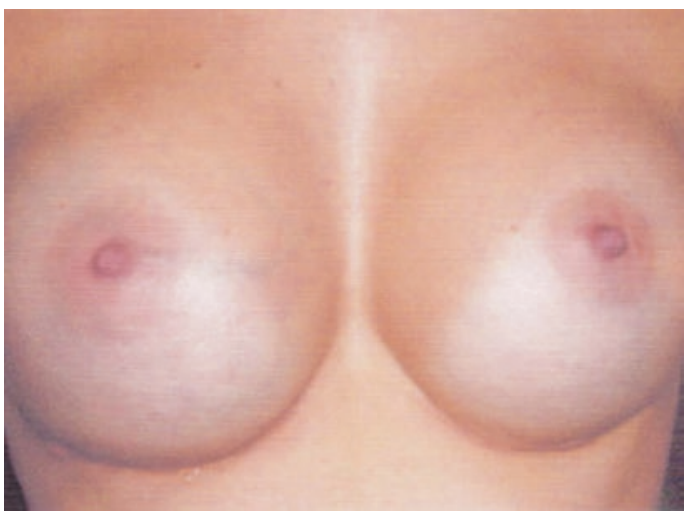

Fig. 148.1 Patient of Dr. Lee who had persistent discharge from the right inframammary wound, starting 1 month after breast augmentation 